\title{
Development of a simple PCR-based assay for the identification of triazine resistance in the noxious plant common ragweed (Ambrosia artemisiifolia) and its applicability in higher plants
}

\author{
Kinga Klára Mátyás · János Taller • \\ András Cseh · Péter Poczai · István Cernák
}

Received: 9 July 2011 / Accepted: 20 July 2011 / Published online: 2 August 2011

(C) Springer Science+Business Media B.V. 2011

\begin{abstract}
Bidirectional allele-specific PCR (BiPASA) was used to detect a point mutation causing triazine resistance in common ragweed (Ambrosia artemisiifolia). The external primers amplified a 278 bp standard DNA fragment in all genotypes. In the susceptible S264S genotypes, a 208 bp fragment was expected while in resistant S264G common
\end{abstract}

Péter Poczai and István Cernák have contributed equally.

Electronic supplementary material The online version of this article (doi:10.1007/s10529-011-0714-5) contains supplementary material, which is available to authorized users.

K. K. Mátyás · J. Taller

Biotechnology Group, Department of Plant Science and Biotechnology, Georgikon Faculty, University of Pannonia, Festetics u. 7, Keszthely 8360, Hungary
A. Cseh
Department of Plant Genetic Resources and Organic Breeding, Agricultural Research Institute of the Hungarian Academy of Sciences, Brunszvik u. 2, Martonvásár 2462, Hungary
P. Poczai $(\square)$
Plant Biology (Biocenter 3), University of Helsinki, PO Box 65, 00014 Helsinki, Finland
e-mail: peter.poczai@gmail.com
I. Cernák $(\square)$
Potato Research Centre, Centre of Agricultural Sciences, University of Pannonia, Festetics u. 7, Keszthely 8360, Hungary
e-mail: i-cernak@ex1.georgikon.hu

ragweed genotypes a $109 \mathrm{bp}$ band was expected. In resistant plants, both the wild and mutant type fragments were detected, indicating that the original triazine sensitive cpDNA is maintained in a heteroplasmic state in the resistant S264G genotypes. Additionally, in silico analysis confirmed the potential applicability of our diagnostic assay for other plant species. In 24 out of 74 taxa (32\%), the assay could be used without any change, while in the others some of the primers should be redesigned before use.

Keywords Heteroplasmy · Invasive plants ·

Ragweed - Weed management · Triazine resistance

\section{Introduction}

Common ragweed, Ambrosia artemisiifolia L. (Asteraceae), is an invasive annual weed native to North America which has become problematic in Europe (Török et al. 2003). It decreases crop yields and represents a major health problem caused by its strongly allergenic pollen (Genton et al. 2005). In chemical plant protection, different triazine herbicides have played a key role in the world's rising crop yields and also in the fight against common ragweed. Because, triazines have a harmful effect on health and environment (Hayes et al. 2010), a decrease in the amount used in those countries where it is approved would be desirable. 
Triazine herbicides inhibit photosynthesis by blocking the electron transfer activity of the D1 protein $(32 \mathrm{kDa})$. The $p s b \mathrm{~A}$ gene, coding the $\mathrm{D} 1$ polypeptide is highly conserved in all photosynthetic organisms (Alfonso et al. 1996); however, an adenine (A) to guanine $(\mathrm{G})$ change was detected previously in the triplet 'TAG' (between the 789-791 bp positions) of the psbA gene (Cheung et al. 1993). This mutated triplet codes glycine (Gly) instead of serine (Ser). This Ser/Gly change occurs at the binding site of the polypeptide and allows the D1 protein to function even in the presence of triazine herbicides.

A wide variety of methods have been developed to identify point mutations and/or single-nucleotide polymorphisms (SNPs). Generally due to their special requirements these techniques are cost-and timeconsuming processes and are not well suited with few SNPs and/or samples. However, PCR allows the application of other simple alternative methods e.g. PCR amplification of specific alleles (PASA) (Sommer et al. 1992). For the PASA reaction a specific primer is designed to perfectly match with one allele but mismatch with the other allele at the $3^{\prime}$ - of the mutation site. This mismatch inhibits the efficient $3^{\prime}$-elongation of the primer by Taq polymerase; therefore the expected DNA fragment is not amplified. Thus, the targeted alleles are detectable based on the presence or absence of the desired fragment(s) after a simple agarose gel electrophoresis. A variant method, namely bidirectional-PASA was also suggested, where one of the alleles is amplified by a PASA reaction in one direction, whereas the second allele is amplified in the opposite direction (Liu et al. 1997).

In the present study, we report the development of a rapid and cost-effective PCR-assay to detect triazine resistance in A. artemisiifolia. We have also tested the applicability of our Bi-PASA method by performing in silico comparisons in other higher plant species.

\section{Materials and methods}

\section{Experimental material}

Forty-eight common ragweed plants-12 triazine resistant and 36 sensitive genotypes - were used. Resistance screening tests were carried out previously by Cseh et al. (2009) according to HRAC criteria for the confirmation of herbicide resistance weeds (Heap 2005).
DNA was purified from $50 \mathrm{mg}$ young common ragweed leaves according to the protocol of Walbot and Warren (1988).

\section{Bi-PASA genotyping}

Primers for the detection of the A to $\mathrm{G}$ nucleotide change were designed based on the sequence of common ragweed $p s b \mathrm{~A}$ gene (DDBJ accession number AB427162). The allele-specific primers were designed according to the finding that a $3^{\prime}$-mismatch does not prime in a PCR at a specific annealing temperature (Sommer et al. 1992). The two external primers $\quad p s b A F \quad\left(5^{\prime}\right.$-ATGAAGGTTACAGATTCG GTC- $\left.3^{\prime}\right), p s b A R$ (5'-AAGGTTAGCACGGTTAATG ATA- $3^{\prime}$ ) were designed based on the findings of Cseh et al. (2009). Two internal primers $B P 1 F$ (5'-GA ACGAGAGTTGTTGAAACC- $\left.3^{\prime}\right)$ and $B P 1 R\left(5^{\prime}\right.$-ATT GATCTTCCAATATGCTA- $3^{\prime}$ ) were designed to specifically prime either to the wild type (A-adenine) or to the mutant type ( $\mathrm{G}-$ guanine) nucleotide.

The PCR amplifications were performed in $10 \mu \mathrm{l}$ containing: $50 \mathrm{ng}$ template DNA, $2 \mathrm{mM}$ dNTP, $1 \mu \mathrm{l}$ $10 \times$ PCR buffer $[(1 \times 10 \mathrm{mM}$ Tris $/ \mathrm{HCl}(\mathrm{pH} 8.8)$, $1.5 \mathrm{mM} \mathrm{MgCl}_{2}, 50 \mathrm{mM} \mathrm{KCl}$ and $0.1 \%$ Triton $\mathrm{X}-100)$ ], 5 pmol of each member of the primer set and 0.25 Unit DynaZyme DNA polymerase enzyme (Finnzymes, Finland). PCR was as follows: 1 cycle $94^{\circ} \mathrm{C}$ for $3 \mathrm{~min}$, followed by 35 cycles of $1 \mathrm{~min}$ at $94^{\circ} \mathrm{C}, 1 \mathrm{~min}$ at the primer annealing temperature and $1 \mathrm{~min}$ at $72^{\circ} \mathrm{C}$. A final extension was applied for $7 \mathrm{~min}$ at $72^{\circ} \mathrm{C}$. The PCR products were separated on $1.5 \%$ agarose gel (Promega, USA) in $0.5 \times \mathrm{TBE}$ (Tris/HCl/boric acid/EDTA) buffer $(220 \mathrm{~V} ; 1.5 \mathrm{~h})$ and post-stained in ethidium bromide.

In silico analysis

We performed a BLAST search using default settings with the sequence of $A$. artemisiifolia psbA gene (AB427162) in GenBank databases (NCBI-http:// www.ncbi.nlm.nih.gov/; DDBJ-http://www.ddbj. nig.ac.jp/). The results of this search, containing 252 entries from different plant species were downloaded in FASTA format. Sequences were aligned with ClustalW implemented in BIOEDIT (Hall 1999) with the default parameters. The data set was further pruned to contain sequences of 74 entries which have relevant agricultural and horticultural importance. 
The complete alignment with further details is provided as Supplementary Table. The pruned dataset was used in the further priming position tests made for each primer across all 74 selected entries to assess the reliability of the designed primers. For these tests $\Delta \mathrm{G}$ values were calculated, as the reliability and/or specificity of the Bi-PASA method depend on the $3^{\prime}$ stability of the primers. This was done because the constancy of a primer can be predicted by calculating the maximum free energy of melting $(\Delta \mathrm{G})$ of the last five bases found at the $3^{\prime}$-end of the primer (Sahdev et al. 2007). An unstable $3^{\prime}$-end (less negative $\Delta \mathrm{G}$ ) will result in less false priming. We calculated the $\Delta \mathrm{G}$ accordingly for each primer across all entries to predict the stability of the designed primers. All calculations were done with the program NetPrimer (http://www.premierbiosoft.com). Resulting values were charted on a Venn-diagram (Fig. 1). $\Delta \mathrm{G}$ values

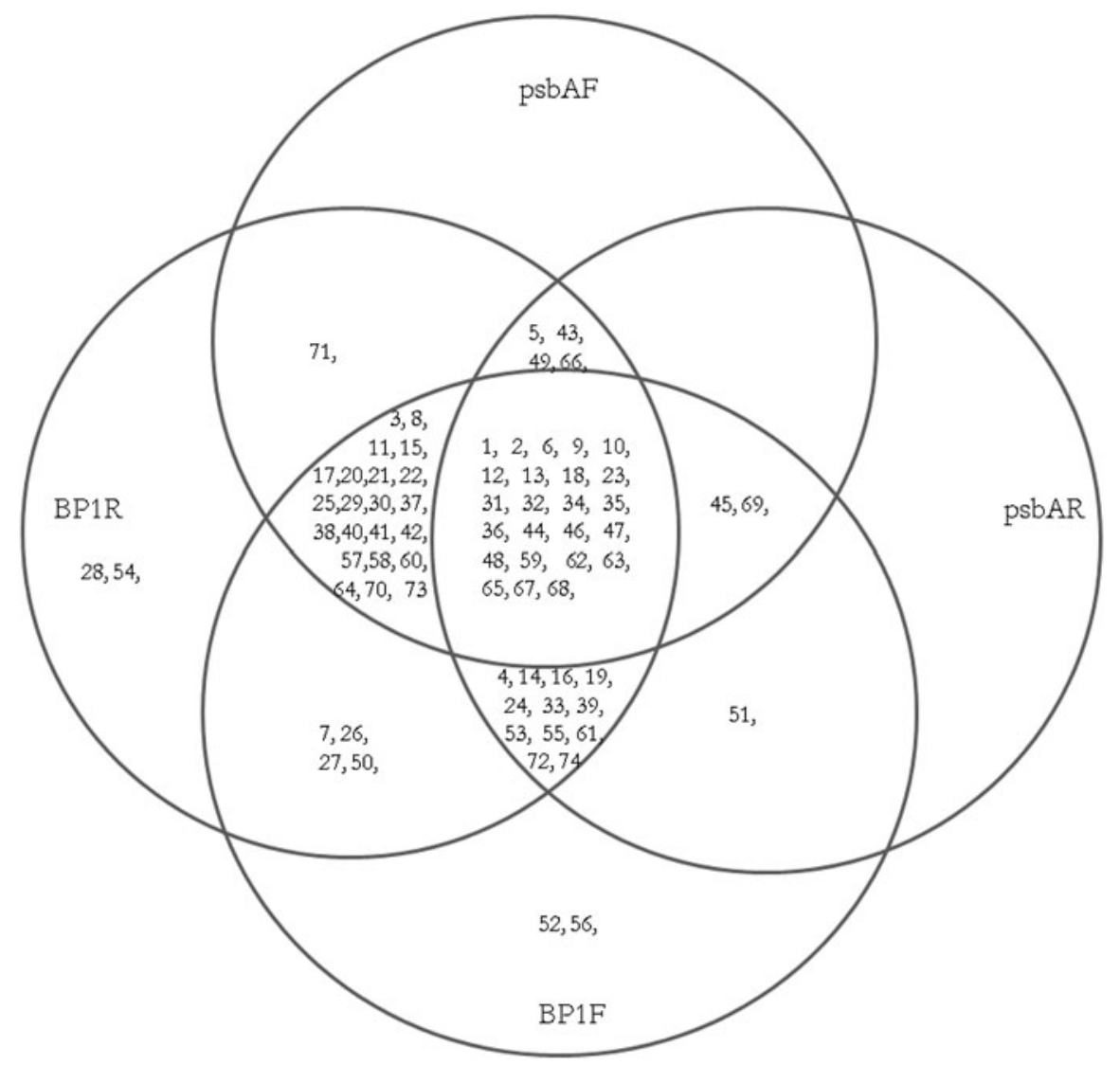

Fig. 1 Summary of the $3^{\prime}$-end stability of the primers in 74 investigated species 1, Ambrosia artemisiifolia; 2, Actinidia deliciosa; 3, Aesculus hippocastanum; 4, Agrostis stolonifera; 5 , Amaranthus powellii; 6, Amaranthus retroflexus; 7, Arabidopsis thaliana; 8, Arabis hirsuta; 9, Atropa bella-donna; 10, Avicennia germinans; 11, Barbarea verna; 12, Bassia scoparia; 13, Beta vulgaris; 14, Brachypodium distachyon; 15, Brassica rapa; 16, Bromus tectorum; 17, Bruguiera gymnorhiza; 18, Ceratophyllum demersum; 19, Chenopodium rubrum; 20, Cicer arietinum; 21, Citrus reticulata; 22, Cucumis sativus; 23, Cuscuta reflexa; 24, Daucus carota; 25, Eucalyptus globulus; 26, Fagopyrum esculentum; 27, Geranium palmatum; 28, Glycine max; 29, Gonystylus bancanus; 30, Gossypium hirsitum; 31, Guizotia abyssinica; 32, Helianthus annuus; 33, Hordeum vulgare; 34, Houttuynia cordata; 35, Jasminum nudiflorum; 36 ,
Lactuca sativa; 37, Lepidium virginicum; 38, Lobularia maritima; 39, Lolium perenne; 40, Manihot esculenta; 41, Medicago sativa; 42, Medicago truncatula; 43, Morus indica; 44, Nicotiana glutinosa; 45, Nicotiana plumbaginifolia; 46, Nicotiana tabacum; 47, Nicotiana tomentosiformis; 48, Nuphar advena; 49, Oenothera biennis; 50, Olimarabidopsis pumila; 51, Oryza sativa; 52, Pelargonium cotyledonis; 53, Phalaris minor; 54, Phaseolus vulgaris; 55, Poa апnиa; 56, Populus alba; 57, Prunus spinulosa; 58, Pyrus communis; 59, Ranunculus macranthus; 60, Raphanus raphanistrum; 61, Saccharatum hybridum; 62, Scaevola aemula; 63, Senecio vulgaris; 64, Sinapis alba; 65, Solanum bulbocastanum; 66, Solanum nigrum; 67, Solanum tuberosum; 68, Spinacia oleracea; 69, Tectona grandis; 70, Trachelium caeruleum; 71, Trifolium subterraneum; 72, Triticum aestivum; 73, Vitis vinifera; 74, Zea mays 

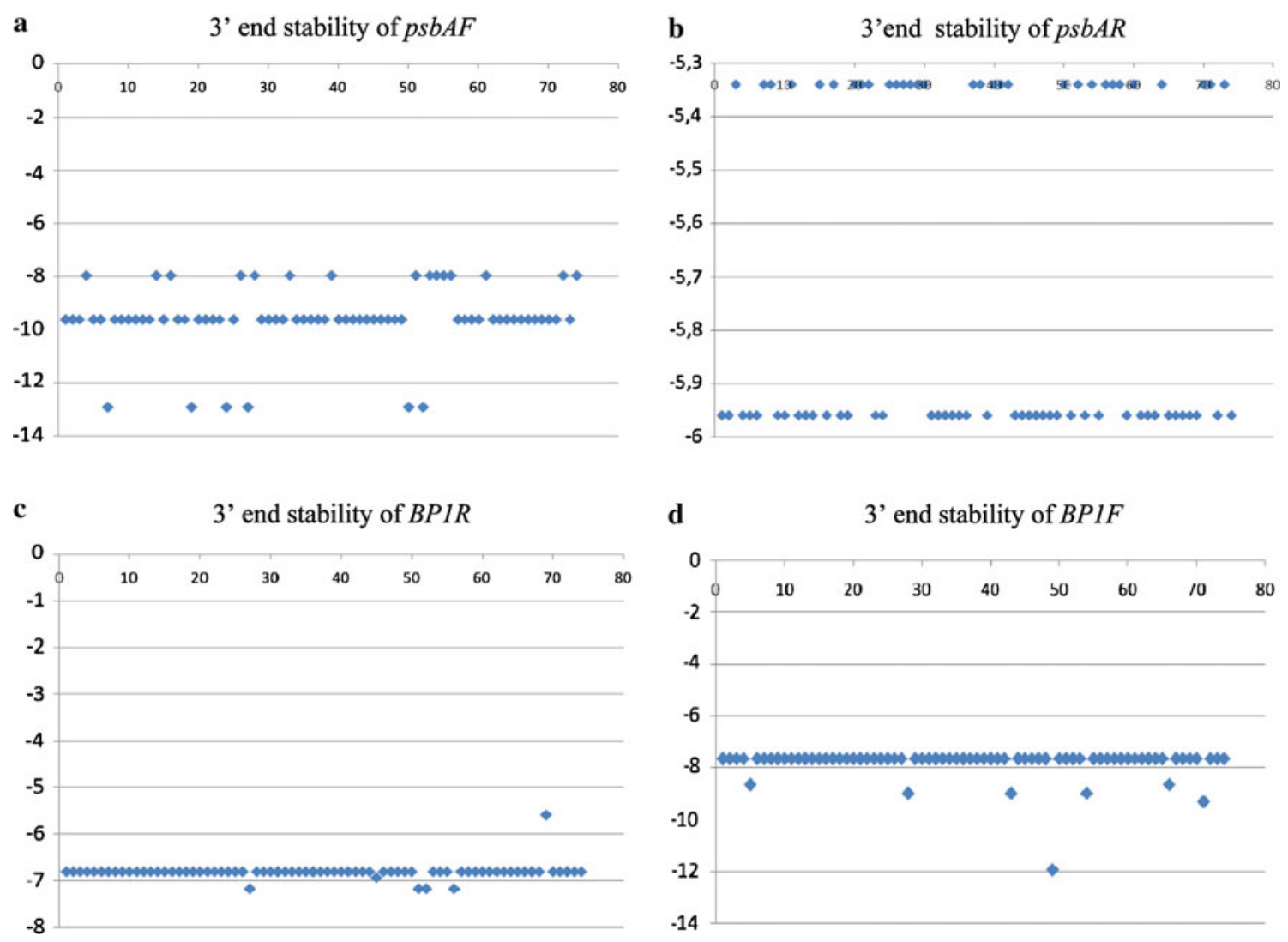

Fig. 2 The $3^{\prime}$-end stability of the primers in different analized species: a $3^{\prime}$-end stability of $p s b A F$, b $3^{\prime}$-end stability of $p s b A R$, c $3^{\prime}$-end stability of $B P 1 R, \mathbf{d} 3^{\prime}$-end stability of $B P 1 F$

of primers developed in this work were used as standards for comparisons among different GenBank entries (Fig. 2a-d). The primers which had substantially distinct $\Delta \mathrm{G}$ values than primers working in A. artemisiifolia were determined as inefficient/ non-usable. The $\Delta \mathrm{G}$ values of common ragweed primers were as follow: psbAF: -9.6 , psabAR: -5.96, BP1F: $-7.65, B P 1 R$ : -6.81 , which served as standard values during primer efficiency tests.

\section{Results}

Test of the primer set

We developed a primer set to detect the triazine resistance causing $\mathrm{A} / \mathrm{G}$ nucleotide change at the $790 \mathrm{bp}$ position of the $p s b \mathrm{~A}$ gene in common ragweed. The amplification patterns of the resistant S264G and susceptible S264S genotypes obtained with the developed primer set are shown in Fig. 3. The primers $p s b A R$ and $p s b A F$ successfully amplified the 278 bp size internal part of the $p s b$ A gene, which contained the mutation point. This product could also serve as an internal positive control, since it should be present in all PCR reactions. The designed external primers amplified two additional bands. In all twelve resistant genotypes which carried a $\mathrm{G}$ nucleotide at the 790 bp position a fragment of 109 bp size was detected, while in the thirty-six susceptible plants (wild biotypes) with an A nucleotide, a $208 \mathrm{bp}$ size fragment (Fig. 3) was amplified. In all resistant biotypes both fragments have been detected indicating that the wild $p s b \mathrm{~A}$ allele is also maintained in heteroplasmic state.

In silico analysis

An in silico analysis was performed to check the utility of our PCR-based detection system in other 
Fig. 3 Schematic representation of the $\mathrm{Bi}$ PASA assay and the amplification patterns of 12 ragweed genotypes. The primers $p s b A F / p s b A R$ amplify a fragment of the psbA gene conferring the point mutation causing atrazine resistance in $A$. artemisiifolia. The primer $B P 1 F$ was designed to specifically anneal to susceptible (wild) genotypes with $A$ at the mutation point, while $B P 1 R$ was designed to amplify only with resistant (mutant) genotypes carrying a $G$ point mutation

\section{Part of the open reading frame of the $p s b A$ gene}

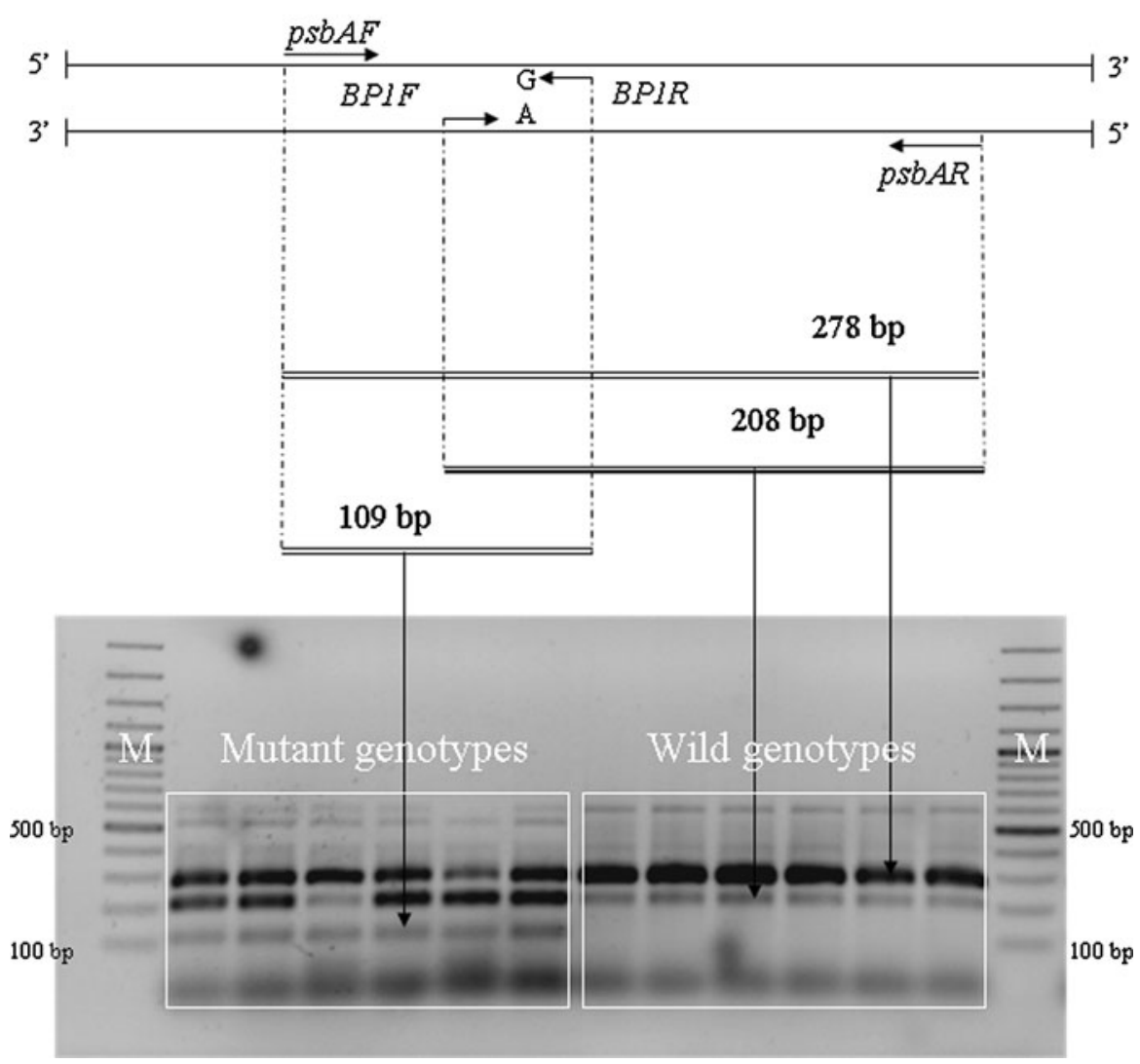

agriculturally relevant plant taxa. Since our diagnostic assay was principally developed for common ragweed, the calculated $\Delta \mathrm{G}$ values obtained for the primer set were used as standards to predict the reliability of the detection system in other plant taxa. Results are given in Fig. 1 while other details are given in supplementary Table 1 . We have found no nucleotide changes in the case of twenty-four analyzed species-names are shown in Fig. 1-at the priming positions of the primer set. We assume that our diagnostic method would be applicable in these taxa based. The results in the case of the remaining species were as follows: in $30 \%$ of the analyzed plant taxa, some nucleotide changes were identified at the priming sites of $p s b A R$, but the remaining three primers of the primer set were able to perfectly anneal at their corresponding position. During sequence alignment and comparison we found four taxa which had some nucleotide change at the priming position of the internal forward primer $(B P 1 F)$ compared to common ragweed. Four species were found where only two internal primers $(B P 1 F$, $B P 1 R)$ may work. Additionally, two plant species were determined where only the internal forward primer $(B P 1 F)$ could anneal; whilst at the priming position of the other three primers, large number of nucleotide changes were revealed. In the case of 12 species only the two internal $(B P 1 F, B P 1 R)$ and the external reverse primer $(p s b A R)$ could anneal reliably at theoretic level. Furthermore, two plant species were found where the two external ( $p s a b A R$, $p s b a R)$ and the forward internal $(B P 1 F)$ primer may work. In two other species only the internal reverse primer $(B P 1 R)$ could anneal. In Oryza sativa only the primers $p s b A F$ and $B P 1 R$ could anneal, whilst in the Trifolium subterraneum the other two primers psbAR and BPIF may work reliably at theoretic level. 


\section{Discussion}

Like other chloroplast genes, $p s b \mathrm{~A}$ is also exceptionally conservative. In various weed and cultivated plant species triazine resistance was found to be caused by a single nucleotide change at the $790 \mathrm{bp}$ position of the $p s b \mathrm{~A}$ gene (Jia et al. 2007; Cseh et al. 2009). Several techniques are applied for the detection of this SNP e.g. restriction digestion with BstXI and MaeI (Thomzik and Hain 1988; Cheung et al. 1993). However, difficulties could appear during the identification with these techniques due to a second MaeI restriction site in many species (Frey et al. 2005) resulting in a more complex restriction pattern. Alternatively, sequencing or quantitative real-time PCR (qPCR) could also be applied, but these are relatively costly and require special expensive instruments. Based on our previous results concerning triazine resistance in common ragweed, here we developed a new PCR-based method (Bi-PASA) for detecting the wild and mutant genotypes in a single PCR reaction even when both chloroplast types are present in the same individual.

To analyze the applicability of the developed BiPASA method, an in silico analysis was performed using sequence information of wild and cultivated species from open databases. Although, the $p s b \mathrm{~A}$ gene of different vascular plants is highly conserved at sequence level ( $>95 \%$ homology) some nucleotide changes at the priming sites of the primer set have been detected. Based on the methodological aspects of our system the positions of these SNPs are very essential. If these occur at the $3^{\prime}$-end of the primer, they could affect successful priming at the corresponding sites. Our results indicated that the PCR-method developed in this study is, theoretically, widely applicable in taxonomically diverse plant species. The developed method could serve as an effective and rapid economical supplement in triazine resistance diagnostics together with dose response assays as such treatments are unable to distinguish homoplasmic and heteroplasmic individuals. Such simple diagnostic methods are useful to study the appearance and frequency of resistance genes in agricultural areas, the major factors controlling gene flow within and between populations and the evolution of resistance genes in populations undergoing different selection pressure. This data can help to develop herbicide rotation strategies essential to prevent the occurrence of resistant weed biotypes.

The collection of genomic data from resistant and susceptible plants in the field allows the recognition of the spread of triazine resistance in an early stage and provides important information to plan effective control strategies in precision agriculture. This data can also help to prevent the development of cross-resistance or multiple-resistance to different herbicides.

In summary, the development of fast and simple bioassays in weed management offer the great potential to carefully plan control strategies based on newly obtained genomic data. As the rate at which plant invasions occur is accelerating globally, such bioassays might have many practical applications in the protection against common ragweed and other noxious weeds.

Acknowledgments This work was supported by the TÁMOP (4.2.2.-08/1/2008-0018) research grant provided for the University of Pannonia. István Cernák gratefully acknowledges the support from the Magyary Zoltan Post-doctoral Fellowship Grant and the EGT/Norway Financial Mechanism. Péter Poczai thanks the support of the Hungarian State Eötvös Research Grant (MÖB/90-1/2011). András Cseh gratefully acknowledges support from the Hungarian Academy of Sciences through the 'Bolyai János' research fellowship.

\section{References}

Alfonso M, Pueyo JJ, Gaddour K, Etienne A-L, Kirilovsky D, Picorel R (1996) Induced new mutation of D1 Serine-268 in soybean photosynthetic cell cultures produced atrazine resistance, increased stability of $\mathrm{S}_{2} \mathrm{Q}_{\mathrm{B}}{ }^{-}$and $\mathrm{S}_{3} \mathrm{Q}_{\mathrm{B}}{ }^{-}$states, and increased sensitivity to light stress. Plant Physiol 112:1499-1508

Cheung W, Cote J, Benoit D, Landry B (1993) A rapid assay for chloroplast-encoded triazine resistance in higher plants. Plant Mol Biol Rep 11:142-155

Cseh A, Cernák I, Taller J (2009) Molecular characterization of atrazine resistance in common ragweed (Ambrosia artemisiifolia L.). J Appl Genet 50:321-327

Frey JE, Frey B, Forcioli D (2005) Quantitative assessment of heteroplasmy levels in Senecio vulgaris chloroplast DNA. Genetica 123:255-261

Genton BJ, Shykoff JA, Giraud T (2005) High genetic diversity in French invasive populations of common ragweed, Ambrosia artemisiifolia, as a result of multiple sources of introduction. Mol Ecol 14:4275-4285

Hall TA (1999) BioEdit: a user-friendly biological sequence alignment editor and analysis program for Windows 95/98/NT. Nucl Acid Symp Ser 41:95-98 
Hayes TB, Khoury V, Narayan A, Nazir M, Park A, Brown T, Adame L, Chan E, Buchholz D, Stueve T, Gallipeau S (2010) Atrazine induces complete feminization and chemical castration male African clawed frogs (Xenopus laevis). PNAS USA 107:4612-4617

Heap IM (2005) Herbicide resistance action committee (HRAC) recommendations for the confirmation of herbicide resistant weeds available at http://www.weedscience.org

Jia X, Yuan J, Shi Y, Song Y, Wang G, Wang T, Li Y (2007) A Ser-Gly substitution in plastid-encoded photosystem II D1 protein is responsible for atrazine resistance in foxtail millet (Setaria italica). Plant Growth Regul 52:81-89

Liu Q, Thorland EC, Heit JA, Sommer SS (1997) Overlapping PCR for bidirectional PCR amplification of specific alleles: a Rapid one-tube method for simultaneously differentiating homozygotes and heterozygotes. Genome Res 7:389-398

Sahdev S, Saini S, Tiwari P, Saxena S, Singh Saini K (2007) Amplification of GC-rich genes by following a combination strategy of primer design, enhancers and modified PCR cycle conditions. Mol Cell Probe 21:303-307

Sommer SS, Groszbar AR, Bottema CDK (1992) PCR amplification of specific alleles (PASA) is a general method for rapidly detecting known single base-pair changes. Biotechniques $12: 82-87$

Thomzik JE, Hain R (1988) Transfer and segregation of triazine tolerant chloroplasts in Brassica napus L. Theor Appl Genet 76(2):165-171

Török K, Botta-Dukat Z, Dancza I, Németh I, Kiss J, Mihaly B, Magyar D (2003) Invasion gateways and corridors in the Carpathian basin: biological invasions in Hungary. Biol Inv 5:349-356

Walbot V, Warren C (1988) Regulation of $M u$ element copy number in maize lines with an active or inactive mutator transposable element system. Mol Gen Genet 211:27-34 